\title{
Practical and Conceptual Analysis of Wingsuit BASE Flight
}

\author{
A. Stöckl', J. Sieker², A. Westman ${ }^{3,4}$, O. Mei-Dan ${ }^{5}$ \\ 1 Praxis für Physiotherapie Stöckl, Unterwössen, Germany \\ 2 San Diego School of Medicine, University of California, San Diego, California \\ 3 Department of Anesthesia and Intensive Care Medicine, Karolinska University Hospital, Huddinge, Sweden \\ ${ }^{4}$ Department of Surgical and Perioperative Sciences, Surgery, Centre for Disaster Medicine, Umeå University, Sweden \\ ${ }^{5}$ Department of Orthopedic Surgery, University of Colorado, Denver, Colorado
}

\section{CORRESPONDING AUTHOR: \\ Jeremy Sieker \\ University of California \\ San Diego School of Medicine \\ 9500 Gilman Drive \\ La Jolla, CA, USA 92093 \\ E-mail: jsieker@ucsd.edu}

DOI:

10.32098/mltj.02.2020.10

LEVEL OF EVIDENCE: 5

\begin{abstract}
SUMMARY
Background. Fixed object parachuting, commonly known as BASE (Building, Antenna, Span, Earth) Jumping, was revolutionized by the introduction of wingsuits in the 1990s. Wingsuit BASE Jumping (WSBJ) has since surged both in overall popularity, and more recently, in its contribution to the rising rate of BASE fatalities. Risks associated with WSBJ and its position within the broader BASE community have been explored in previous work. However, the practical considerations of a nominal wingsuit flight, the aerodynamic underpinnings of WSBJ, and discussions regarding the pilot's decision-making processes and in-flight goals are nearly absent from the current literature.

Methods. This expert opinion article was developed through years of experience in the BASE environment and analysis of in-flight altimetry and glide data from both the authors and through contributors in the wingsuit BASE community. Previous authors' rigorous investigations and thorough work on safe, high-performance WSBJ are also discussed.

Results. This concept article takes a very practical approach to WSBJ, walking through the optimal procedure for a safe jump from exit to parachute deployment.

Conclusions. Strong conceptual foundation, focus on technique, lessons from relevant accidents, and emergency planning all contribute to a successful wingsuit BASE jump.
\end{abstract}

\section{KEY WORDS}

Wingsuit, BASE, jumping, proximity, parachutist

\section{INTRODUCTION}

The advent of modern wingsuits has profoundly changed the landscape of BASE (Building, Antennae, Span, Earth) fixed object jumping. In addition to the skill sets required for skydiving and non-wingsuit BASE jumping (nWSBJ), wingsuiting BASE jumping (WSBJ) requires significant experience with wingsuit equipment, wingsuit flight, and an understanding of how the suit can influence each component of a BASE jump.

Available data clearly indicates that BASE fatalities associated with wingsuiting are representing an increasing proportion of annual BASE fatalities over the last 20 years (1-3). It is not currently known whether WSBJ is associated with a different rate of non-fatal accidents and injuries than nWSBJ. What is known is that BASE jumping injuries are most often orthopedic, with the lower limbs being the most commonly affected anatomic region (4). The rate of severe injuries in BASE, defined as those requiring recovery periods of 21 or more days, has been estimated to be 1 in 500 jumps, with less severe injuries occurring in 1 of 250 jumps $(1,5)$.

In the time between the first BASE fatality in 1981 and the first wingsuit BASE fatality in 2001, errors associated with glide miscalculation and deployment timing only represented $11.5 \%$ of fatal BASE accidents, and no fatalities were attributed to vertical object face freefall collisions (3). From 2002-2018, since the first wingsuit BASE fatality, 30.1\% of BASE fatalities are attributable to glide miscalculation or deployment timing and $5.6 \%$ to vertical object face freefall collisions (3). Wingsuits were involved in a large number of 
these fatalities and may be at least partially responsible for the overall shift in fatality risk profile across the jump.

Beyond previous discussions of injury rates, fatality rates, and some work on the psychology of wingsuit BASE jumping, there is relatively little information in peer-reviewed literature on the topic of wingsuit BASE jumping (6). However, many non-academic sources, from books to blogs, are generated from within the WSBJ community on various topics within WSBJ (7-9). The purpose of this paper is to discuss ways to improve in-flight performance, explore methods of mitigating the risks associated with WSBJ, and to improve the conceptual and practical understanding of what WSBJ requires of the pilot for researchers, jumpers, and enthusiasts alike. To organize the discussion, this will be accomplished in a stepwise format that mirrors the progression of tasks in a wingsuit BASE jump.

\section{METHODS}

This phase-based understanding of wingsuit flight reflects expert opinion on the practice of WSBJ. It was developed through years of experience in the BASE environment and analysis of in-flight altimetry and glide data from both the authors and contributors in the WSBJ community. Previous authors' rigorous investigations and thorough work on safe, high-performance WSBJ were invaluable in the refinement of these ideas (7-10). This discussion is intended specifically for topics of WSBJ, and some principles may not be constructive or valid for the purposes BASE jumps conducted without wingsuits.

The methods used for the preparation of this article are not regulated by the United States, German, Israeli or Swedish legislation regarding research on humans. The authors took into account ethical issues that may have appeared through pursuing its preparation.

\section{RESULTS: STAGE-BASED ANALYSIS OF A WINGSUIT BASE JUMP}

\section{Phase 1: the exit}

The first phase of flight is composed of the initial jump from the object and the initial moments of freefall. It presents its challenges in three major ways. First, other than exceptional cases of proximity WSBJ, this is the phase of flight in which the jumper is closest to the object, increasing the risk of potentially deadly vertical or horizontal terrain strikes. Second, is the phase of flight that corresponds to the lowest total airspeed experienced in the jump. Aerodynamic control in freefall depends heavily on strong, consistent airflow, so this segment of low total airspeed flight is partic- ularly problematic. It is for this reason that a precise exit is crucial for a successful BASE jump. Third, while most components of a BASE jump can be practiced in the relatively controlled skydiving environment, it is not currently practical for the majority of BASE jumpers to achieve a high degree of practice in zero airspeed exits in the skydiving environment. Zero airspeed skydive exits, typically performed from hot air balloons and helicopters, are very expensive and are imperfect analogues, given that the exit point is not as rigid as most BASE objects would be. In addition, they can require awkward exit stances unlike those of the BASE environment.

Importantly, very few BASE objects, such as unusually high bridges, offer a "safe" way to train the full wingsuit exit, including transition to stationary glide. These factors make wingsuit exits very difficult to train safely. It is therefore important for all wingsuit BASE jumpers to understand proper exit procedure thoroughly, as it is their main tool for quickly entering controlled flight and gaining separation from the exit object as soon as possible.

Speaking practically, three main components must be controlled for a successful exit: pushing power, pushing direction and rotation speed. The ideal footing for a wingsuit BASE exit is that of a symmetric, balanced jump from a dry, clean, sturdy object with either a vertical or inclined surface. The two-leg jump is considered to be more stable and more powerful than a single-leg jump (11). This is a kind of counter-movement jump used to achieve maximum height, but is essentially performed 'sideways' in the BASE environment. There are instances where a staggered singleleg jump can provide initial momentum that proves beneficial for certain exit points. Experienced, well-rounded jumpers will be capable of, and comfortable with, both styles. Whichever one chooses, the priorities of a strong push for exit separation, symmetry and balance must be maintained. Inclined footing, or horizontal footing with a vertical edge, is preferred because it allows the jumper to make a strong horizontal push with minimal risk of slipping, which would be more likely to occur from a horizontal force exerted on a horizontal object (figure 1). It is also preferred for the tips of the jumper's shoes to reach over the edge, if one exists,

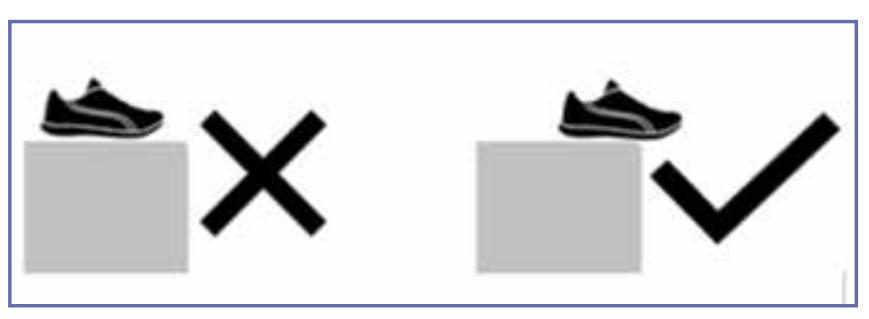

Figure 1. Preferred footing for BASE exits.

Muscles, Ligaments and Tendons Journal 2020;10 (2) 
to prevent slips off the object, which are a common catastrophic error (3).

An exit's power, direction and rate of rotation have significant influence on control and stability in early flight. In the initial fall, trajectory and pitch are largely ballistic. Little aerodynamic control is available, so the focus is on making a strong push and controlling the forward rotation speed (figure 2). The ideal pitch for a wingsuit jump at the moment of exit is horizontal $\left(0^{\circ}\right)$, ending with controlled rotation towards a pitch of $-45^{\circ}$ at the initial moments of useful aerodynamic control.

The importance of the strength and direction of the jumper's push quickly become apparent. Some jumpers are tempted by, or may simply find it difficult to avoid, the addition of a vertical component to their exit push. However, in addi- tion to complicating pitch control, a "head high" push with an initial positive vertical speed reduces horizontal separation from the object (figure 4). Jumpers should not attempt to jump upwards. New jumpers are commonly taught to keep their "eyes on the horizon" on exit. This is correct in that it indicates horizontal movement as the priority, but is misguided in that there can be a large difference between where a jumper is looking and how their body is oriented. The phrase "push at the horizon!" may therefore be a more accurate and useful approach in WSBJ. This way of thinking about the exit helps keep the initial pitch horizontal $\left(0^{\circ}\right)$. While the push is meant to be perfectly horizontal, wingsuit jumpers are more often instructed to look about $-45^{\circ}$, to avoid being "head-high", a common problem associated with looking at the horizon during exit.

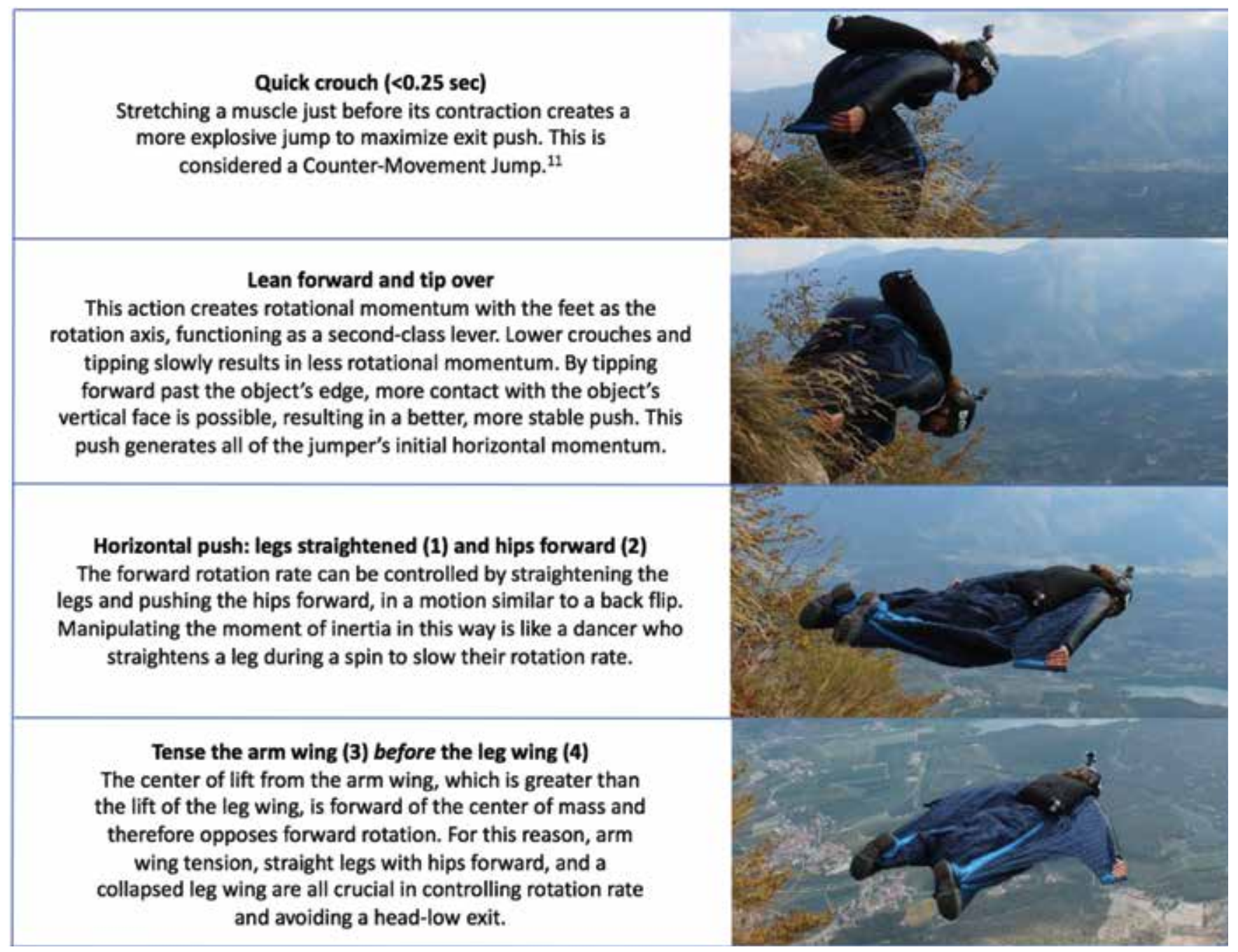

Figure 2. Wingsuit BASE exit procedure (Picture: Lino Oehl). 


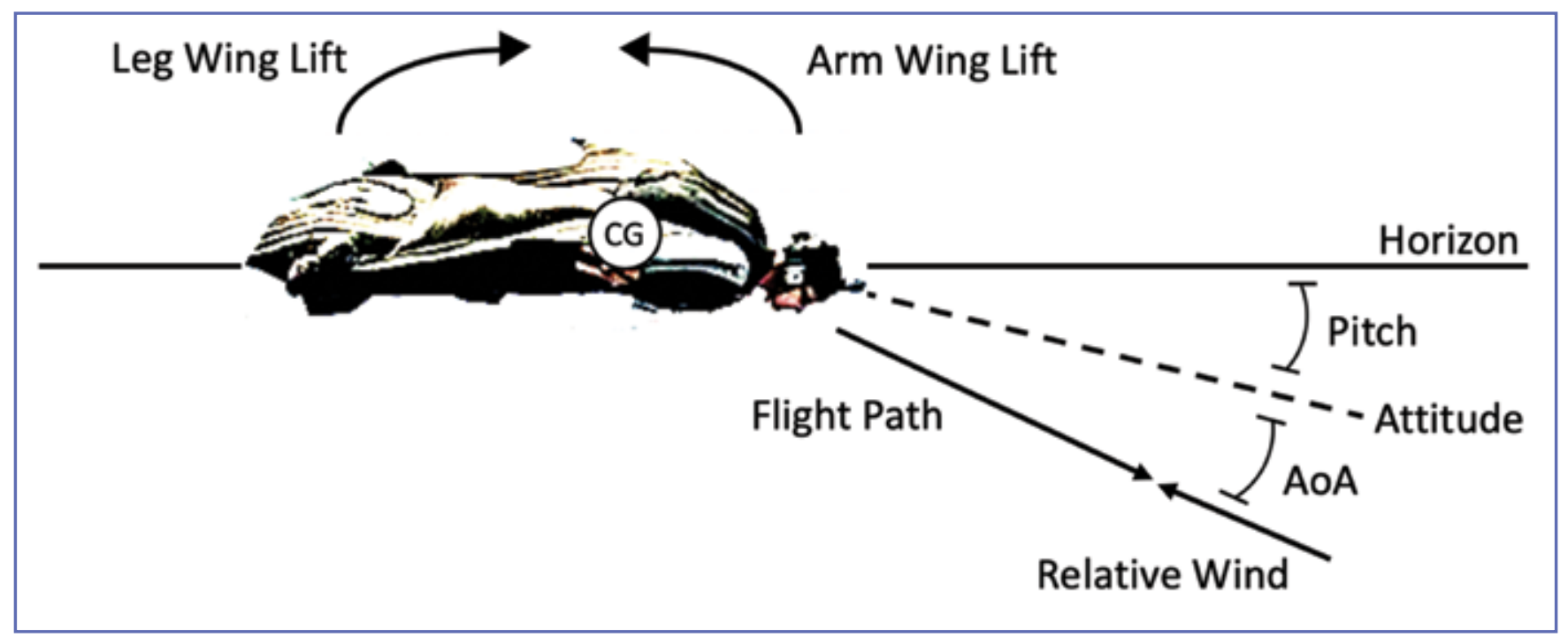

Figure 3. Wingsuit free-body diagram (CG = Center of Gravity, AoA = Angle of Attack, adapted from TopGun BASE10 and Robson and D'Andrea 201012, photo from the personal collection of Author J.S.)

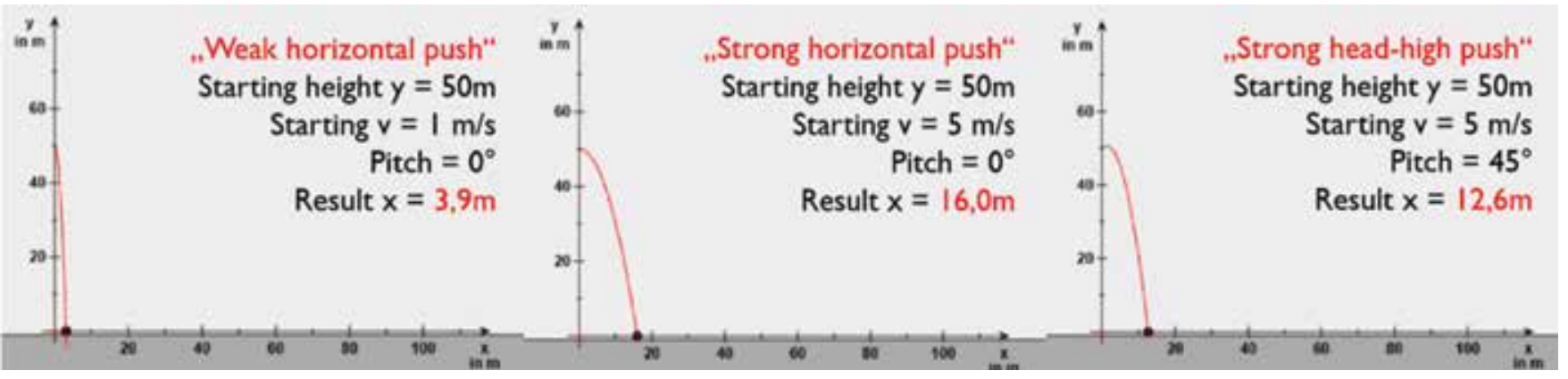

Figure 4. Projected ballistic trajectories and corresponding exit parameters.

Using widely available GPS- and altimetry-based flight equipment, jumpers can analyze their own exit performance. The ideal exit is one with maximized horizontal speed and vertical speed near zero at the moment of exit. Speaking practically, in analysis of one's glide data, a good push will create a horizontal speed greater than the initial vertical speed. The better the push, the farther apart those two speeds will be.

Some exit points are "underhung", meaning that some lower altitude portion of the object extends horizontally beyond the exit point itself in the same direction of the jumper's initial push. On an object like this, falling straight down from the exit point would either be impossible or would guarantee object impact. A strong exit is imperative to avoid object collision, as there are many fatal incidents due at least in part to a weak exit.
The theoretical scenario for these accidents is as follows. A wingsuit BASE jumper at an underhung object performs an uncharacteristically weak exit. This leads to insufficient horizontal object separation and a collision with the object almost immediately after jumping. The force of impact makes establishing aerodynamic stability impossible and the jumper fatally impacts the ground or the cliff a second time soon after. Unfortunately, this scenario has been rather common in WSBJ.

\section{Phase 2: The Start}

'The Start' is the period of the initial dive from the establishment of $-45^{\circ}$ pitch until the flattening of pitch and the establishment of glide. As the exit phase closes, minimum total airspeed has developed in the initial freefall to estab- 


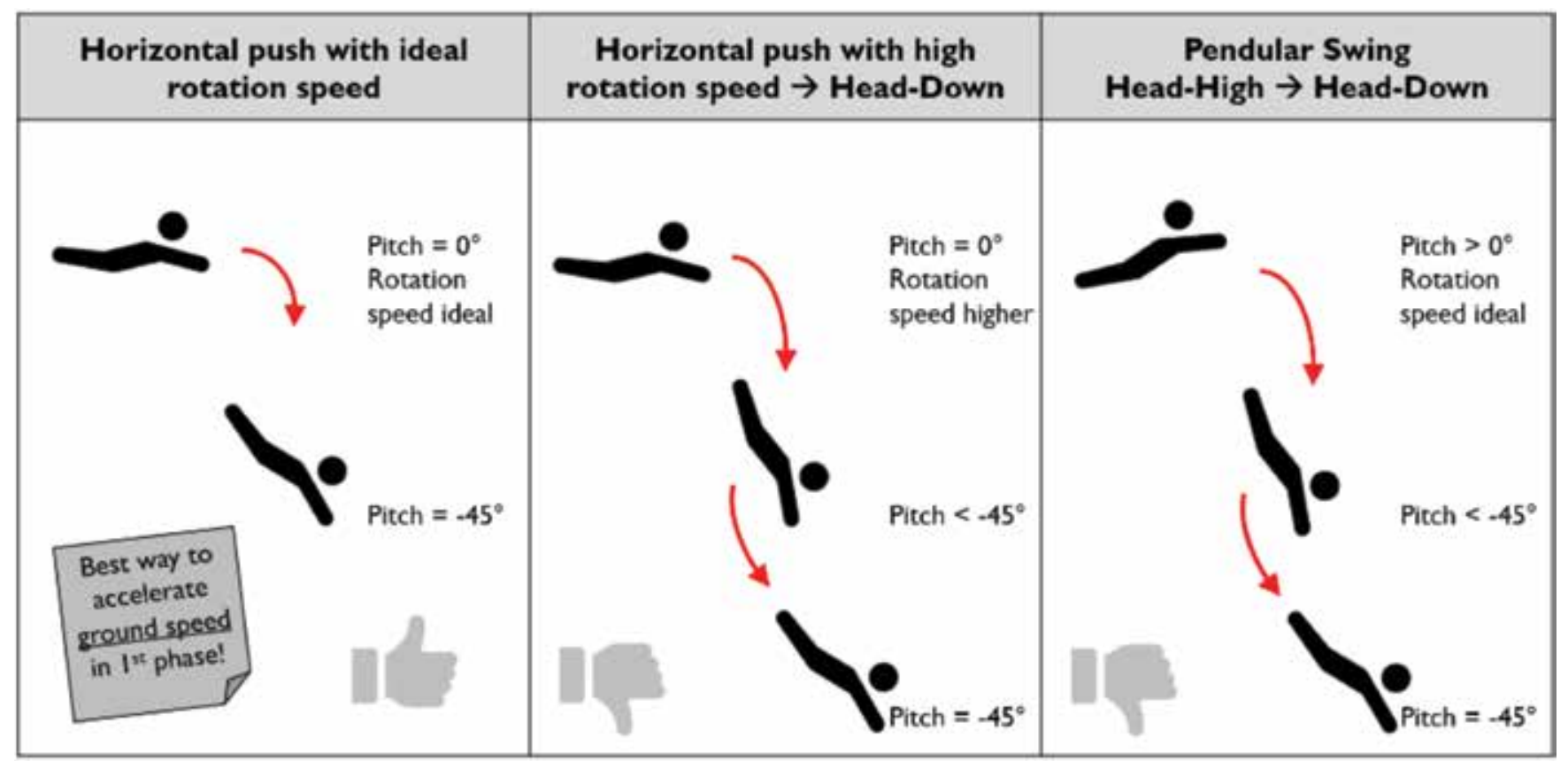

Figure 5. Pitch variation and object separation concept.

lish aerodynamic control. At this point, the decision can be made to recover quickly and establish the stationary glide ratio as soon as possible, known as the short exit, or to continue diving in order to continue building speed, known as the race exit (figures 6, 7). Whichever the pilot chooses, the suit's initial aerodynamic stall transitions to glide and tension is applied to the leg wing. The pilot's pitch, under- stood as angle between the wingsuit's chord line and the horizon (figure 3), is maintained constant at $-45^{\circ}$ throughout the second phase. In contrast, as the horizontal airspeed continues to increase, the angle of attack (AoA) slowly decreases as the relative wind shifts from being vertical (from directly below) to horizontal. For a short exit, this phase is typically around $0.5-1.5$ seconds, but is maintained

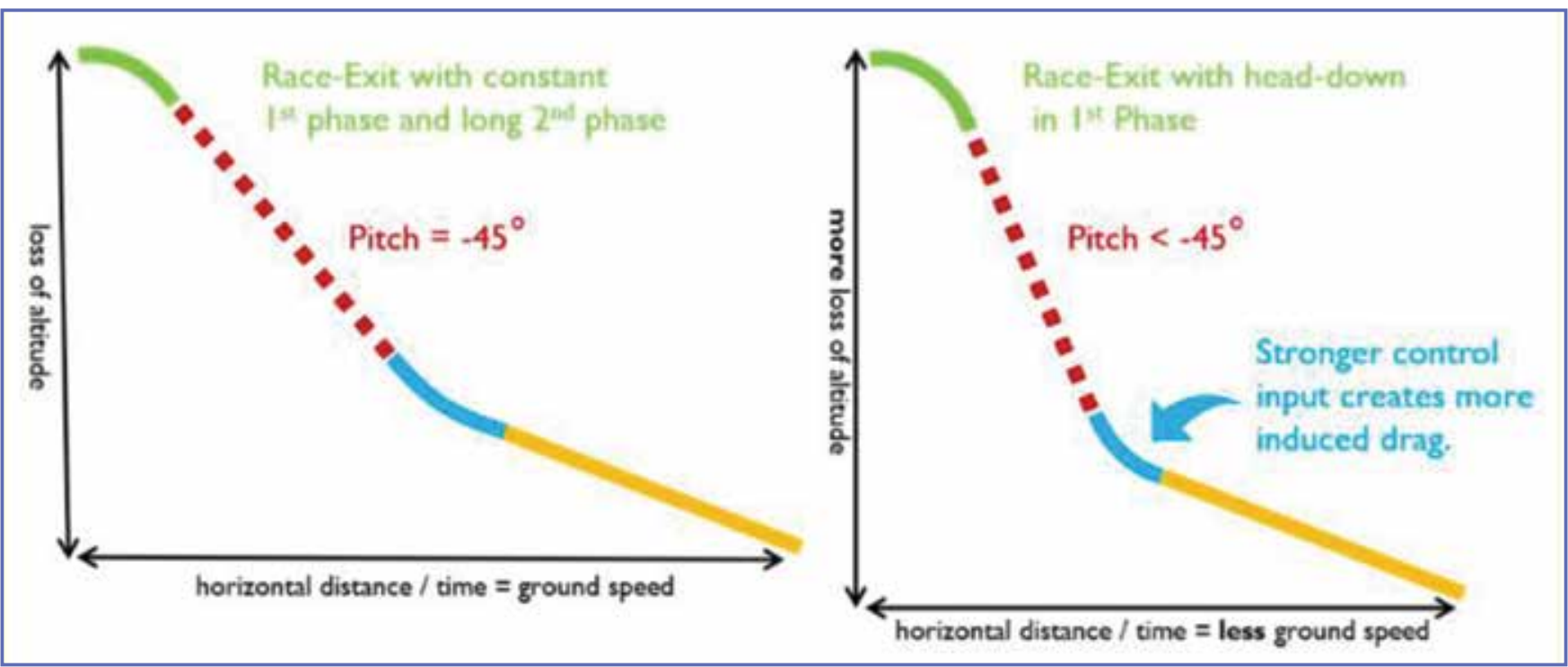

Figure 6. Race exits and exit orientation conceptual diagram. 
for up to 10 seconds in some race exits (figure 8). However, these are only general descriptors. Specific time intervals must be re-evaluated based on the goals and needs of each jump and jumper.

While all exits are meant to be safe, some jumpers elect to follow different strategies for different jumps. Jumpers usually employ the short exit to achieve maximum horizontal glide with the smallest possible loss of altitude. This ultimately conserves energy and maximizes glide capacity later in the jump. In contrast, the race exit is a procedure intended to maximize groundspeed at the expense of glide. Some jumpers believe that by exiting "head low", with a higher forward rotation speed and end-exit pitch below $-45^{\circ}$, they can improve their race exits. Robust data do not yet exist on this subject, but the theory behind this approach may be misguided for three reasons. The first two have to do with safety, and the third being that this procedure may in fact obstruct the race exit's primary goal, maximizing groundspeed.

First, a dive pitch below $-45^{\circ}$ compromises one's ability to adequately separate from the object, creating a safety hazard. Secondly, by training this exit, one risks interfering with muscle memory for a normal maximum-separation exit. Cases do exist where the habit of performing race exits on every jump has led to jumpers unintentionally using this procedure at objects requiring short exits (unpublished communication, Amrei Stöckl and Lino Oehl). Third, while the decreased initial AoA provided by a head-low exit (pitch $<-45^{\circ}$ ) does cause a quicker increase in airspeed through reduced drag, one must consider that this reduced AoA also requires higher airspeed to produce useful lift when compared to a normal-pitch exit, which compromis- es horizontal acceleration. Just because an exit achieves high airspeed more quickly does not necessarily mean a net increase of ground speed exists across the jump, which is the goal of the race exit. In this way, an initial pitch $<-45^{\circ}$ may be self-defeating in horizontal speed outcomes in addition to compromised safety, as previously mentioned.

In terrain proximity flight, exits with pitch $<-45^{\circ}$ are sometimes performed with the intention of achieving aerodynamic control as quickly as possible. This shares similar problems as when a race exit is performed head-low (pitch $<-45^{\circ}$ ). The safety problems are similar, but also include compromised long-term glide in the case of proximity flights with a "flatter" overall glide profile. Regarding performance, it must again be considered that a steeper pitch (pitch $<-45^{\circ}$ ) results in a smaller AoA in early flight, which requires a higher total airspeed to be useful than the same wing at a higher AoA, up to the Ao $A_{\text {STALL }}$. This steep exit pitch may result in delayed aerodynamic authority. However, steep pitch $\left(<-45^{\circ}\right)$ may be entirely proper in proximity flight after aerodynamic control is attained, given the unique goals and safety procedures of terrain proximity flight.

\section{Phase 3: Glide Transition}

'Glide Transition' represents the transition from a dive to the stationary glide phase of flight. By the end of this phase, the total airspeed is constant, and acceleration has stopped. By recovering from a dive, the pitch becomes more horizontal. However, because the horizontal speed is increasing, the AoA does not meaningfully change until the target glide speed is reached. Imprecise or aggressive control inputs and

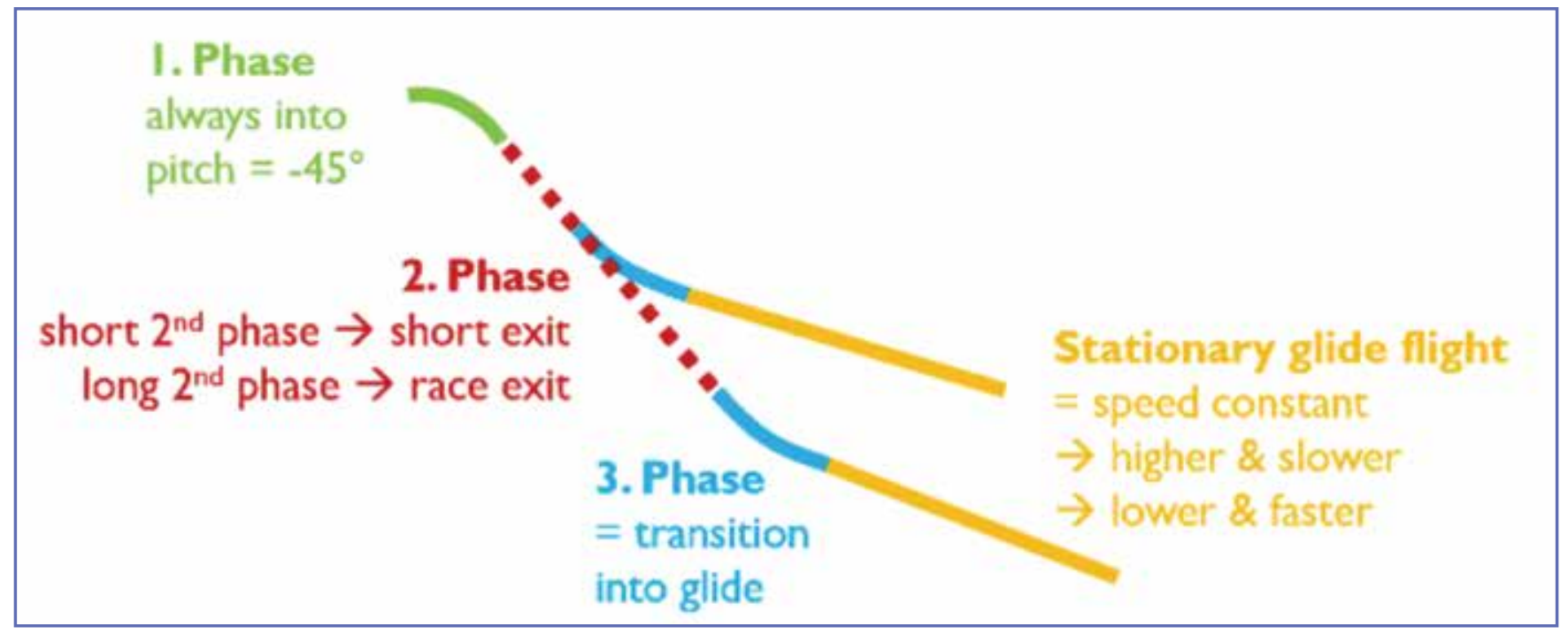

Figure 7. Flight Phase illustration. 
body position result in energy loss to drag and reduced glide capacity-common issues in this phase.

These initial three phases of wingsuit flight require skill sets that are quite different from those of non-wingsuit BASE jumps. In early BASE training, jumpers learn to jump "head-high", with a pitch at or above the horizon (pitch > $0^{\circ}$ ). This muscle memory must be overwritten to properly execute the horizontal push, pitch control and AoA control necessary for WSBJ.

A way to visualize these initial phases is to imagine a $-45^{\circ}$ reference line coming down and out from the exit point. The point at which a jumper's glide path passes this imaginary reference line can be understood to represent their "start-arc" (8). While this metric does not account for total airspeed attained at that moment, it can be one way to understand exit performance, when properly contextualized. A sample of altitude and GPS tracking data in short and race exits is available in figure $\mathbf{8}$. When improving exits, jumpers are often tempted to buy bigger and more advanced wingsuits in order to artificially inflate their abilities. However, better theoretical understanding, education, and experience are much stronger steps towards the safe, proper development of a BASE jumper than using equipment to fill skill gaps.

It is important to define what is meant by a "short exit", given that it may refer to the quickest crossing of the $-45^{\circ}$ line or the least amount of altitude lost at some other time point. Stated simply, the duration of phase 2 and the desired speed to reach during phase 3 must be chosen carefully based on exactly where in the flight profile the altitude loss

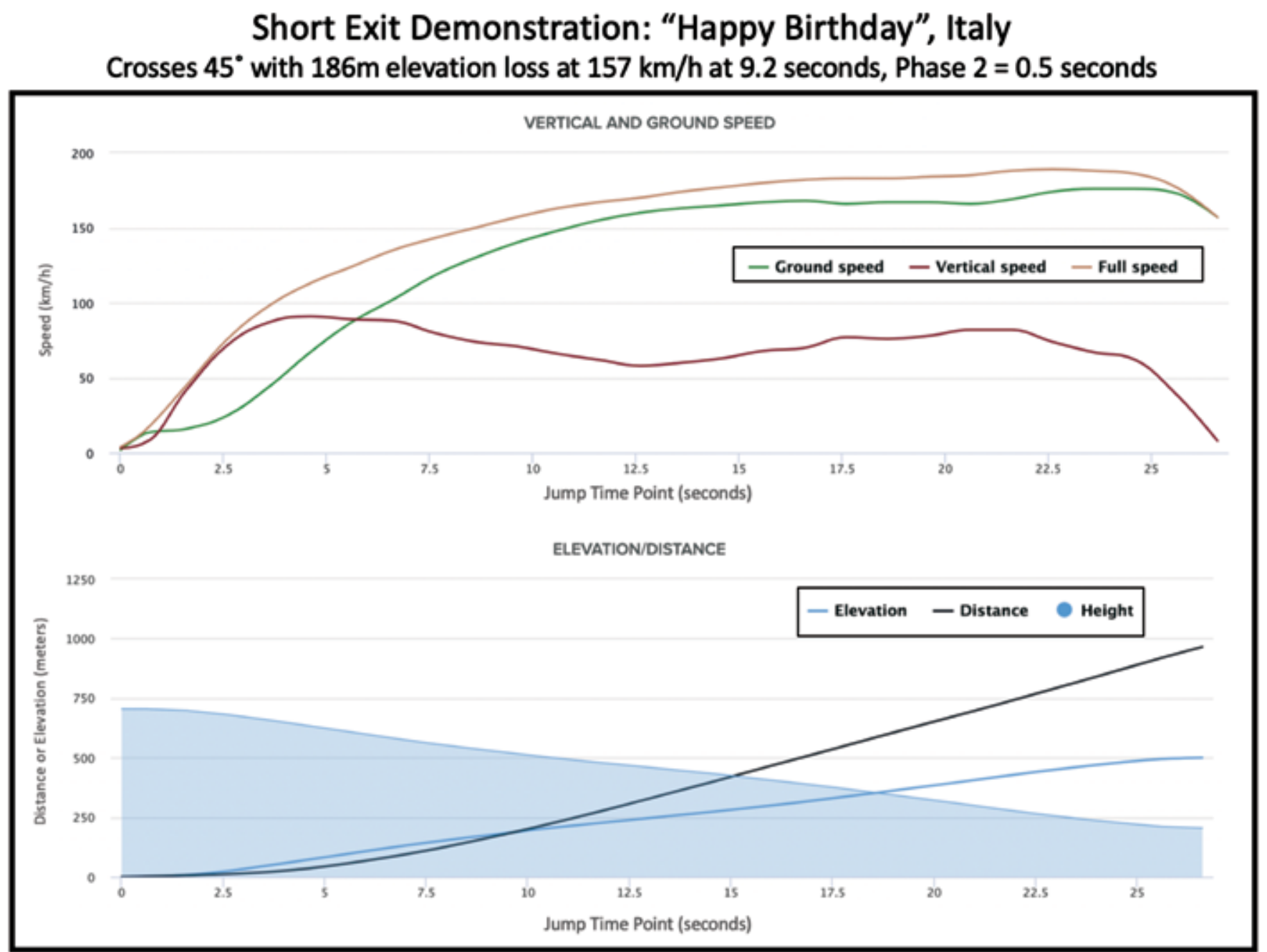

Figure 8. Sample glide data for each exit type (Same pilot [Lino Oehl], same wingsuit [Colugo 3], same first phase exit procedure, and comparable weather conditions). 


\section{Race Exit Demonstration: Monte Brento, Italy Crosses $45^{\circ}$ with $620 \mathrm{~m}$ elevation loss at $250 \mathrm{~km} / \mathrm{h}$ at 17.7 seconds, Phase $2=8.0$ seconds}

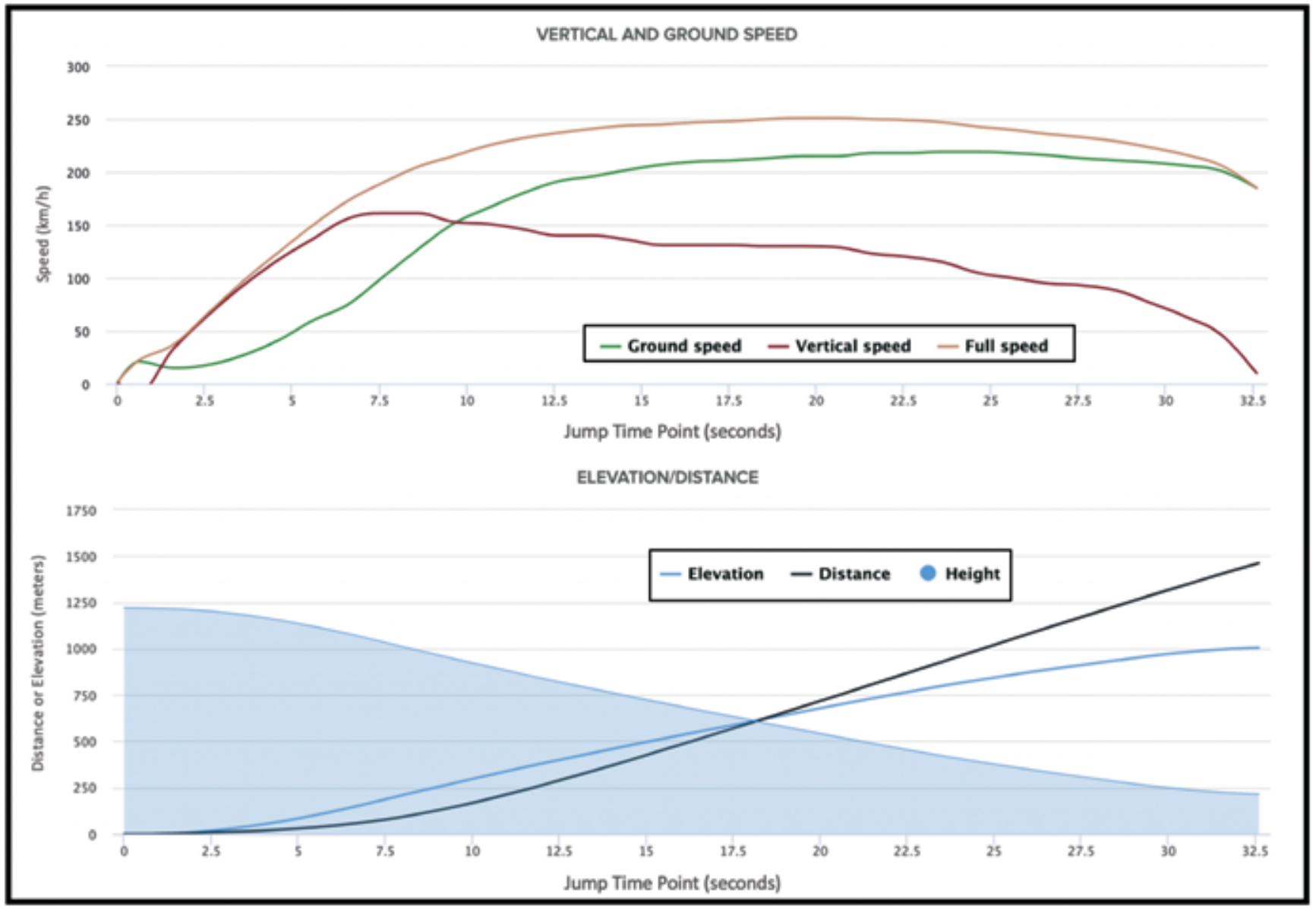

must be minimized. The best start arc is typically achieved when phase 2 ends at the suit's minimum airspeed for flight. This minimizes altitude loss at the crossing of $45^{\circ}$ from the exit point, but does not provide the best glide for the jump as a whole. This is because the speed at the end of phase 2, in that case, would be less than the suit's best glide speed. To maximize glide at any distance beyond the start arc, phase 2 needs to end at the suit's airspeed of best glide. The tradeoff between these two types of altitude-saving measures must be navigated with care based on the glide goal for and risks presented by each jump. The start arc is not the whole picture when it comes to glide and total airspeed needs to be accounted for in the discussion of short starts.

A good exit is the appropriate use of potential energy for that object and context based on glide, speed, and safety goals, with aerodynamic control starting as soon as possible. For these reasons, a start arc does not completely capture the notion of a short exit or the overall quality of any given start. The definition of good performance on a wingsuit flight always depends on the jumper's goals and the demands of the object.

\section{Phase 4: Stationary Glide Maintenance}

The stationary glide phase of a WS BASE jump extends from the establishment of glide speed to the beginning of deployment or the pre-deployment flare. This phase typically occupies the largest portion of time spent during a WSBJ. The stationary glide phase requires a continual, careful analysis of one's resources (altitude, speed, alternative routes), the challenges presented by the ongoing jump, and the adequacy of those resources to meet the evolving challenges. Familiarity with the terrain and slope, the altitude necessary to safely cross each region of potential flight, knowl- 


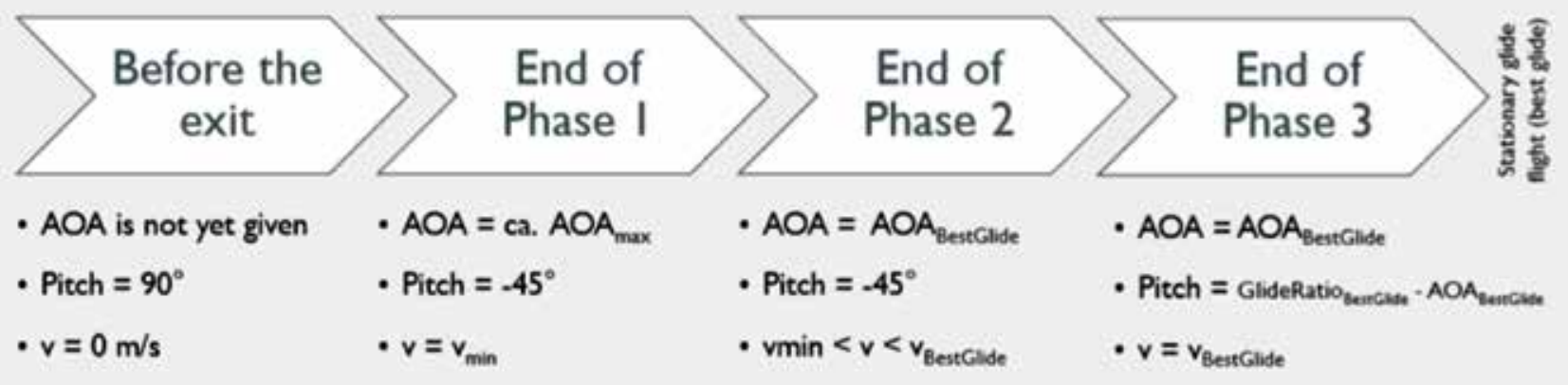

Figure 9. Aerodynamic stage definitions for shortest possible exit at best glide speed (AOA = Angle of Attack, $v=V e l o c i t y$, $\mathrm{ca}=$ approximately).

edge of available landing areas, and local weather expertise are among the knowledge that must be established before a jump can even be considered. Then, during the flight, a wingsuit pilot must be experienced with estimating their glide, relative altitude, and airspeed adequacy by feel, with or without a functioning glide monitor. These two data sets, resources and risks, must then be compared moment-bymoment, with decisions being made by instinct and experience, given the lack of time for contemplative thought. In these ways, pre-flight planning and preparedness are crucial for success in stationary glide (figure 9).

Emergency routes are key components of a thorough pre-flight plan. Commonly known as "outs," these alternative routes typically improve safety by allowing for more immediate altitude loss than the initially-selected route. Any number of other factors can lead to a situation where the primary route is no longer possible, such as inadequate altitude, insufficient airspeed, equipment problems, or medical issues, among others. A good "out" typically allows the jumper to safely regain airspeed and terrain separation, or at the very least provides a different route to a safer landing area. Casually acknowledging that a different route exists is not adequate. Understanding the outs' characteristics, terrain descent profile, where they lead, if they have any landing areas, their weather considerations, and how one can communicate with their group after landing are among the factors that must be well understood before relying on any route, primary or otherwise.

In more advanced jumps, there are likely to be sections of the planned jump where no outs exist. Theoretically, the safest option is to avoid jumps where this is the case. However, if one chooses to undertake these jumps, steps can still be taken to improve safety. Any section with no outs should be entered with as much speed and spare altitude as possible, to build margin for unexpected energy losses or imper- fect glide maintenance. In addition, a landmark before the final out should be identified at which the jumper positively confirms to themselves that they securely have the altitude and airspeed to successfully navigate the following section. The purpose of this landmark is similar to that of the "decision altitude" in skydiving. It is a point past which the emergency route is automatically taken unless the primary option can confidently be employed. Lastly, at all times, but particularly in sections with no out, jumpers should be mentally prepared for a "panic pull," an immediate emergency parachute deployment in a suboptimal time or location, at any point. If a jumper's glide is worsening, terrain clearance is decreasing, or the jumper is noticing themselves slowing down or pitching up more than normal to maintain altitude, parachute deployment before the situation deteriorates any further is reasonable. Obviously, this is not ideal, but many fatalities and injuries have occurred as a result of waiting too long for an emergency deployment.

In proximity flight, jumpers choose geographic routes shaped to allow flight very close to the object for some period of time. A route's specific combination of geography and flown altitude can be referred to as a "line". High airspeed and proper line selection are crucial to success in proximity flight. A jumper with a consistently high airspeed can pitch up or flare at any moment to quickly gain separation from the object. Jumpers new to proximity flight are often taught to select lines that require them to intentionally dive, such that only intentional movements could force them further down. Anything other than purposefully diving harder would cause them to flatten their glide and separate from the terrain because of the high airspeed. Training of this sort on steep terrain reduces the likelihood of collisions and trains the habit of diving to terrain, rather than pitching up and hoping that one's airspeed will be adequate to clear the object. 
As a matter of principle, subtle movements, stability, and well-refined body position will allow the maximum conversion of altitude into speed, rather than losses to drag or exaggerated adjustments in body position.

In training for proximity flight, jumpers will often fly the same route multiple times, reducing object separation slightly with each successive attempt. This represents a series of nearly identical lines, differing only in altitude. In this type of training, altitude should be lost in a way that contributes to airspeed (through diving), rather than by flying in an intentionally inefficient way or through maneuvers such as "s-turns". In addition, the jumper should only continue on a new line with less object separation when they are at peak performance on that jump. Going lower, or even at the same level of proximity, with an imperfect exit, recovery, or glide may diminish more safety margin than the jumper's experience can tolerate. Even without noticing it, the jumper may be flying a higher AoA to hold the same line, which may not be recoverable. A comparably safe way to train this is by use of a variable inclination wind tunnel (13, figure 10).

There are many WSBJ fatalities attributable to glide miscalculation. Interestingly, there are at least three cases in which wingsuit BASE jumpers have survived terrain collision that occurred due to glide miscalculation. In each of these, the jumper did not have the altitude or airspeed required to fly the selected line. The classic progression of this accident is that a jumper will commit to a line with no outs, notice that they are lower than they planned and therefore, consciously or otherwise, pitch up to regain the lost altitude. This pitch adjustment helps maintain altitude momentarily, but costs airspeed. Eventually their airspeed runs out, the angle of attack increases past the stall point, and aerodynamic lift is lost, resulting in terrain collision. Situational awareness, selecting lines appropriate for one's performance and experience level, and actively considering "outs" are the simplest solutions to this problem.

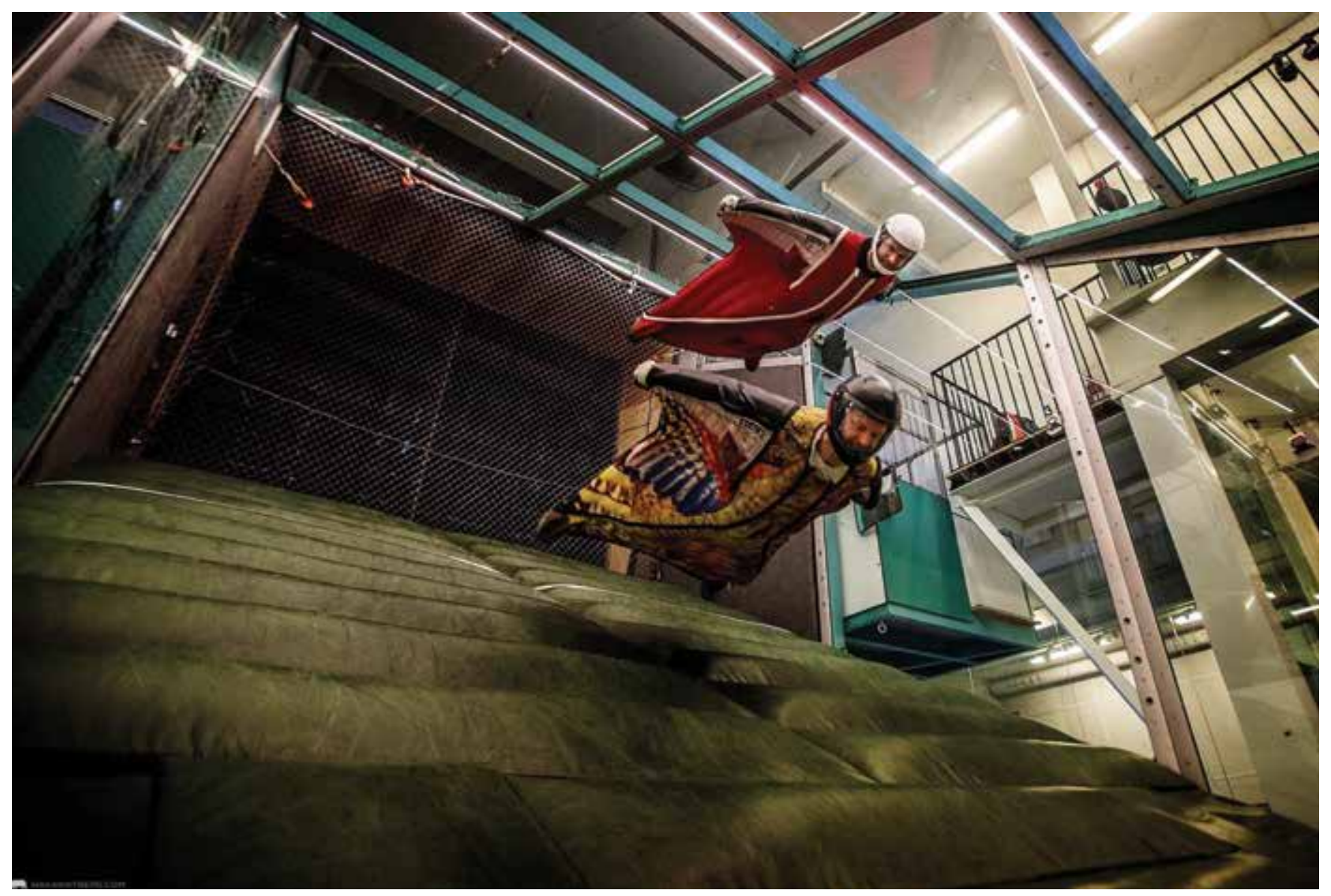

Figure 10. Wingsuit pilots training in an inclined wind tunnel (Stockholm, Sweden) having a variable inclination range that covers the stationary glide performance of current wingsuit models. Take-off and landing are from/to the floor, meaning that only Phase 4 can be realistically trained. Photo courtesy of Espen Fadnes and Håkan Nyberg. 


\section{Phase 5: Flare}

Before deployment, it is possible to convert kinetic energy from horizontal and vertical speed back into altitude with a maneuver called a flare. This procedure allows for deployment at a slightly higher altitude and lower airspeed, which improves jumper safety. During a flare, the angle of attack is increased, slowing vertical fall rate, increasing horizontal speed temporarily, causing a short gain in altitude, and ending with a decrease in total airspeed. Put simply, kinetic energy from the vertical fall rate is converted into horizontal speed through increased AoA. Kinetic energy from this high horizontal speed is then converted into potential energy from increased altitude as the horizontal speed deteriorates in the climb.

This maneuver is not simple and must be practiced in the skydiving environment before it can be executed well in WSBJ. It requires precise execution because it can involve controlled flight at both very high and very low airspeeds, both of which can make a wingsuit difficult to control immediately before deployment. In addition, because this maneuver is used at the end of a jump, an asymmetric or unstable flare typically cannot be repeated due to altitude restriction. A common misconception is that long, high speed dives are necessary for a strong flare. However, effective flares can be conducted without such dramatic expenditure of altitude, and some degree of flare is possible even from the relatively low speed of best glide. ${ }^{8}$ If flaring from a very high airspeed, the rate of AoA change must be carefully controlled to avoid a high-speed stall and may not be more effective in regaining altitude than if the maneuver were initiated from Phase 4 airspeed.

\section{Phase 6: Canopy Deployment}

Canopy deployment presents many risks to BASE jumpers. From equipment failures to malfunctions and opening in a direction different than the direction of flight (off-heading), there are many ways that deployment can quickly create dire problems for BASE jumpers. In addition, the problem of limb confinement within the wingsuit can amplify these issues for wingsuiters, who may not be able to operate their primary canopy flight controls for a number of seconds after deployment.

\section{REFERENCES}

1. Mei-Dan O, Carmont MR, Monasterio E. The epidemiology of severe and catastrophic injuries in BASE jumping. Clinical Journal of Sport Medicine: Official Journal of the Canadian Academy of Sport Medicine 2012; 22(3): 262-267. https://doi.org/10.1097/JSM.0b013e31824bd53a
For these reasons, a history of consistent, on-heading deployments in nWSBJ and wingsuit skydiving are absolutely necessary before transitioning to WSBJ.

Many WSBJ fatalities are associated with technical errors at deployment. Two common avoidable issues are "missed pulls," when the jumper is not able to find the pilot chute to begin deployment, and catastrophic flight instability occurring because of asymmetric, stalled, or prolonged deployment procedures. Familiarity with one's BASE equipment, currency of flight experience, responsible packing, vigilant gear inspection and maintenance, and well-practiced deployment technique can all help avoid these issues at deployment. Lastly, as previously mentioned, emergency deployments ("panic pulls") can lead to dangerous landing situations, but if it becomes clear that a jumper lacks the airspeed, altitude, or stability to successfully complete the flight with adequate safety margin, it may be in their best interest to deploy sooner rather than later. Jumpers must always be mentally prepared to do it.

\section{SUMMARY}

An exit with a strong horizontal push and controlled rotation to a dive with pitch of $-45^{\circ}$, reached at minimum airspeed of aerodynamic control, is ideal (Phase 1). Ballistic rotation can be controlled up to this point by straightening one's legs, pushing the hips forward, and tensing the arm wing. This dive (Phase 2) is maintained at a pitch of $-45^{\circ}$ for a duration of time suited to the goal and requirements of the individual jump. Jumpers then use gentle control inputs to establish their speed and AoA based on the goals of the jump (Phase 3). The AoA is held constant during Phase 3, which finishes when the speed of best glide is reached. This glide speed and AoA are then maintained for the duration of the jump, or adjusted as appropriate per the jump's goals, with constant vigilance regarding one's altitude, speed, and available outs (Phase 4). A flare can then be conducted to slow one's airspeed and regain altitude temporarily (Phase 5). The jump then concludes with a smooth, controlled, symmetric deployment (Phase 6).

\section{CONFLICT OF INTERESTS}

The authors declare that they have no conflict of interests (14).

2. Mei-Dan O, Monasterio E, Carmont M. Westman, A. Fatalities in Wingsuit BASE Jumping. Wilderness \& Environmental Medicine 2013; 24 (4): 321-327. https://doi. org/10.1016/j.wem.2013.06.010. 
3. Sieker JW, Castillo EM, Vilke, GM. Timing of fatal BASE jumping incidents: 1981-2018. Journal of Forensic and Legal Medicine 2019. https://doi.org/10.1016/j.jflm.2019.04.011.

4. Monasterio E, Mei-Dan O. Risk and severity of injury in a population of BASE jumpers. The New Zealand Medical Journal 2008; 121(1277): 70-75.

5. Soreide K, Ellingsen CL, Knutson V. How dangerous is BASE jumping? An analysis of adverse events in 20,850 jumps from the Kjerag Massif, Norway. The Journal of Trauma 2007; 62(5): 1113-1117. https://doi.org/10.1097/01. ta.0000239815.73858.88.

6. Holmbom M, Brymer E, Schweitzer RD. Transformations through Proximity Flying: A Phenomenological Investigation. Frontiers in Psychology 2017; 8. https://doi.org/10.3389/ fpsyg.2017.01831.

7. Gerdes M. (n.d.), Wingsuit Nerding | Squirrel; retrieved June 22, 2019, from https://squirrel.ws/nerds.

8. Gerdes, M. 2014. The great book of BASE. BirdBrain Publishing.
9. Webb, R. (n.d.). BLOG - Topgunbase; retrieved June 22, 2019, from http://topgunbase.ws/news/.

10. Wingsuit Aerodynamics: Angle of Attack (Part 1) - YouTube. (n.d.); retrieved June 22, 2019, from https://www.youtube. $\mathrm{com} /$ watch? $=4 \mathrm{BOVi9fQhQw \& t=297s}$.

11. Ham DJ, Knez WL, Young WB. A deterministic model of the vertical jump: implications for training. Journal of Strength and Conditioning Research 2007; 21(3): 967-972. https://doi. org/10.1519/R-16764.1.

12. Robson G, D'Andrea R. Longitudinal stability analysis of a jet-powered wingsuit. In AIAA Atmospheric Flight Mechanics Conference 2010 (n.d.).

13. Westman A, Georén P, Strömberg J. Inclined Wind Tunnel for the Study of Human and Large Animal Flight. Wilderness Environ Med. 2017; 28(1):54-56.

14. Padulo J, Oliva F, Frizziero A, Maffulli N. Muscles, Ligaments and Tendons Journal - Basic principles and recommendations in clinical and field Science Research: 2018 update. MLTJ 2018; 8(3): $305-307$. 